OPEN ACCESS

Edited by:

Karen Moreau,

Université de Lyon, France

Reviewed by:

Lhousseine Touqui,

Institut Pasteur, France

Paul Briaud,

Ohio University, United States

${ }^{*}$ Correspondence:

Friedrich Götz

friedrich.goetz@uni-tuebingen.de

Specialty section:

This article was submitted to

Molecular Bacterial Pathogenesis,

a section of the journal

Frontiers in Cellular and

Infection Microbiology

Received: 28 November 2021

Accepted: 16 December 2021

Published: 06 January 2022

Citation:

Biswas L and Götz F (2022)

Molecular Mechanisms of

Staphylococcus and Pseudomonas Interactions in Cystic Fibrosis.

Front. Cell. Infect. Microbiol. 11:824042.

doi: 10.3389/fcimb.2021.824042

\section{Molecular Mechanisms of Staphylococcus and Pseudomonas Interactions in Cystic Fibrosis}

\author{
Lalitha Biswas ${ }^{1}$ and Friedrich Götz ${ }^{2 *}$ \\ ${ }^{1}$ Centre for Nanosciences and Molecular Medicine, Amrita Vishwa Vidyapeetham, Kochi, India, ${ }^{2}$ Microbial Genetics, \\ Interfaculty Institute of Microbiology and Infection Medicine Tübingen (IMIT), University of Tübingen, Tübingen, Germany
}

Cystic fibrosis (CF) is an autosomal recessive genetic disorder that is characterized by recurrent and chronic infections of the lung predominantly by the opportunistic pathogens, Gram-positive Staphylococcus aureus and Gram-negative Pseudomonas aeruginosa. While $S$. aureus is the main colonizing bacteria of the CF lungs during infancy and early childhood, its incidence declines thereafter and infections by $P$. aeruginosa become more prominent with increasing age. The competitive and cooperative interactions exhibited by these two pathogens influence their survival, antibiotic susceptibility, persistence and, consequently the disease progression. For instance, $P$. aeruginosa secretes small respiratory inhibitors like hydrogen cyanide, pyocyanin and quinoline $\mathrm{N}$-oxides that block the electron transport pathway and suppress the growth of S. aureus. However, S. aureus survives this respiratory attack by adapting to respirationdefective small colony variant (SCV) phenotype. SCVs cause persistent and recurrent infections and are also resistant to antibiotics, especially aminoglycosides, antifolate antibiotics, and to host antimicrobial peptides such as LL-37, human $\beta$-defensin (HBD) 2 and HBD3; and lactoferricin B. The interaction between $P$. aeruginosa and $S$. aureus is multifaceted. In mucoid $P$. aeruginosa strains, siderophores and rhamnolipids are downregulated thus enhancing the survival of $S$. aureus. Conversely, protein A from $S$. aureus inhibits $P$. aeruginosa biofilm formation while protecting both $P$. aeruginosa and $S$. aureus from phagocytosis by neutrophils. This review attempts to summarize the current understanding of the molecular mechanisms that drive the competitive and cooperative interactions between $S$. aureus and $P$. aeruginosa in the CF lungs that could influence the disease outcome.

Keywords: cystic fibrosis, Staphylococcus aureus, Pseudomonas aeruginosa, antagonism, adaptation, co-existence

Abbreviations: AHL, $\mathrm{N}$-acylhomoserine lactone; AQNOs, alkyl-hydroxyquinoline N-oxides; CF, cystic fibrosis; CFTR, transmembrane conductance regulator; ETC, electron transport chain; HBD, human $\beta$-defensin; HCN, hydrogen cyanide; HQNO, 2-heptyl-4-quinolone N-oxide; PYO, pyocyanin; PQS, Pseudomonas quinolone signal; ROS, reactive oxygen species; SCV, small colony variant; ASL, airway surface liquid. 


\section{INTRODUCTION}

Cystic fibrosis (CF) is an inherited autosomal recessive genetic disorder that predominantly affects Caucasians. CF is caused due to the mutations in the cystic fibrosis transmembrane conductance regulator (CFTR) gene. The CFTR gene is present on the long arm of the chromosome 7 at the position $7 \mathrm{q} 31.2$. It has 27 exons and codes for the 1480 amino acid containing CFTR protein that functions as a voltage gated chloride ion channel that transports chloride ions into and out of the cells. The CFTR protein is found across the membranes of epithelial cells that line the liver, lungs, pancreas, intestines, skin and reproductive tract. CFTR in general coordinates the rate of sodium ion $\left(\mathrm{Na}^{+}\right)$absorption and chloride ion $\left(\mathrm{Cl}^{-}\right)$secretion to hydrate airway surfaces by effecting the osmotic movement of water and promotes mucus clearance (Anderson et al., 1991). To date $>1000$ mutations have been reported in the CFTR gene. The most common of these mutations that accounts for $\sim 50-70 \%$ of $\mathrm{CF}$ cases worldwide is the in-frame deletion of phenyl alanine at position $508(\Delta \mathrm{F} 508)$. CFTR that lacks phenyl alanine at position 508 gets misfolded and fails to get translocated to the cell membrane and will get degraded by the quality-control mechanism of the cell (Debraekeleer and Daigneault, 1992; Bobadilla et al., 2002). In CF, the absence of a functional CFTR in the cell membranes upsets the $\mathrm{Na}^{+}$and $\mathrm{Cl}^{-}$ion balance that is required for maintaining the normal, hydrated thin mucus layer that can be easily cleared by cilia lining the lungs and other organs. The $\mathrm{Na}^{+}$and $\mathrm{Cl}^{-}$ion imbalance causes retention of water inside the cells (Anderson et al., 1991). The consequent dehydration of the extracellular space creates a thick, viscous mucus layer that cannot be cleared easily by cilia and traps bacteria resulting in chronic infections. The main characteristic feature of $\mathrm{CF}$ is the chronic bacterial infections of the airways and sinuses.

As shown by the Cystic fibrosis foundation, patient registry, 2020 annual data report that lungs of the CF patients get colonized and infected by multiple bacteria throughout life and that the bacterial flora in the lung changes as individuals age (CysticFibrosisFoundation, 2020). More than $60 \%$ of patients harbor at least one microorganism even at very young ages, and this increases to more than $80 \%$ in older age groups. S. aureus is the most common microorganism across all age groups and as patients' age it is commonly found together with $P$. aeruginosa. However, with increasing age the incidence of $S$. aureus infections declines slightly and infections with $P$. aeruginosa become equivalent or even more prominent (Figure 1). In addition to these two pathogens, the CF lungs were also found to be infected to a lesser extent by Burkholderia cepacia, Haemophilus influenzae, Stenotrophomonas maltophilia and Achromobacter xylosoxidans (Lyczak et al., 2002; Lipuma, 2010).

Whereas in the majority of healthy individuals, Prevotella, Veillonella, Staphylococcus, Streptococcus, Corynebacterium, Fusobacterium, and Propionibacterium were the typical genera found in the upper respiratory tract while $P$. aeruginosa rarely infects healthy human lungs (Williams et al., 2010; Clark, 2020).Exaggerated pro-inflammatory response following bacterial infections and the consequent respiratory failure are the main causes of morbidity and mortality in CF. Interactions among these bacteria greatly influence the lung function. During the early stages of infection, $P$. aeruginosa outcompetes $S$. aureus by secreting virulence products that inhibit the growth and induce the lysis of $S$. aureus. However, $S$. aureus still survives and persists in the presence of $P$. aeruginosa by employing several strategies. Several host factors have also been reported to influence the coexistence of $S$. aureus and $P$. aeruginosa. This review provides an overview of the interactions involving initial competitive, adaption and later co-operative interactions between $S$. aureus and $P$. aeruginosa, the two most common bacterial pathogens that inhabit the CF lungs.

\section{TOXINS OF $P$. aeruginosa THAT INHIBIT S. aureus GROWTH}

$P$. aeruginosa produces a large number of virulence factors that influence the severity of CF disease. The expression of approximately $10 \%$ of the $P$. aeruginosa genome including the genes for various virulence factors and biofilm formation is dependent on its cell density-based quorum sensing (QS) systems, LasI/R, RhlI/R and Pseudomonas quinolone signal (PQS) systems. The LasI/R, Rhli/R cell-to-cell communication systems consists of enzymes involved in the synthesis of diffusible autoinducer $\mathrm{N}$-acylhomoserine lactone (AHL) signal molecules and a target regulator to monitor the density of the population (Pearson et al., 2000). The PQS system involves the quinolone QS signals, 4-hydroxy-2-heptylquinoline (HHQ) and 2-heptyl-3,4-dihydroxyquinoline (PQS, pseudomonas quinolone signal (Deziel et al., 2004). With increasing cell density, these diffusible signaling molecules would accumulate and trigger coordinated responses in $P$. aeruginosa (Van Delden and Iglewski, 1998). In the LasI/R QS system, LasI directs the synthesis of $\mathrm{N}$-(3-oxododecanoyl)-l-homoserine lactone (3oxo-C12-HSL) for LasR activation, and in the Rhl system, RhlI, directs the production of $N$-butanoyl-L-homoserine lactone (C4-HSL) for the activation of RhlR. The QS signals of the PQS system involves derivatives of 4-hydroxy-2alkylquinolines (HAQs) including the derivatives of HHQ and PQS that are synthesized by the enzymes PqsABCDE and PhnAB and lead to the activation of PqsR. The LasR-3-oxo-C12-HSL complex positively regulates the transcription of RhlI and RhlR. Both the Las and Rhl QS systems control the production of PQS molecules, $\mathrm{PQS}$ in turn regulates the expression of RhlI and RhlR (Juhas et al., 2005). Activation of these QS systems in $P$. aeruginosa leads to the production of several virulence factors such as ADP-ribosyltransferase toxins (exotoxin A and exoenzyme S), quinoline $\mathrm{N}$-oxides, siderophores (pyochelin, pyoverdine), mucoid exopolysaccharide (alginate), endotoxin (lipopolysaccharide, LPS), phenazine pigment pyocyanin, proteases (LasA, LasB, LecA, LecB type IV protease, alkaline, and protease IV), phospholipase A2 (ExoU), and rhamnolipid etc. A number of these virulence factors that are produced by $P$. aeruginosa including the respiratory toxins (hydrogen cyanide (HCN), pyocyanin and quinoline $\mathrm{N}$-oxides), LasA, 
Prevalence of Respiratory Microorganisms by Age Cohort, 2020

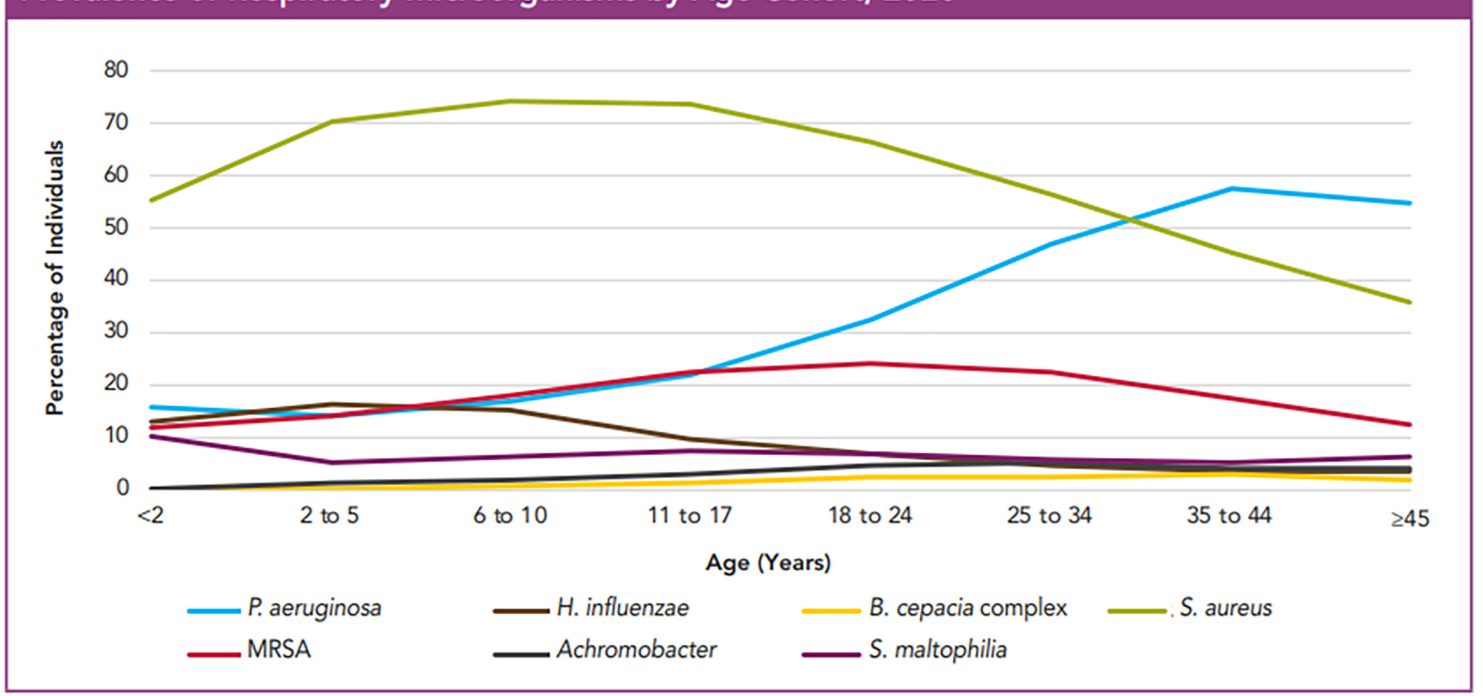

FIGURE 1 | Prevalence of Respiratory Microorganisms by age cohort, 2020. The figure shows the prevalence of microorganisms in the respiratory tract of CF patients in different age groups. Even at a very young age, affected individuals have at least one microorganism, and this number increases with increasing age. $S$. aureus is by far the most widespread microorganism, which is followed with time by $P$. aeruginosa. What one can also see is that the incidence of MRSA is only about $20 \%$ of that of MSSA. Hemophilus influencae, Achromobacter, Burkholderia cepacia and Stenotrophomonas maltophilia were only rarely observed. This image is reproduced with permission from Cystic Fibrosis Foundation Patient Registry, 2020 Annual Data Report, Bethesda, Maryland ${ }^{\circledR} 2021$ Cystic Fibrosis Foundation.

rhamnolipids, long chain $\mathrm{N}$-acyl homoserine lactones (AHLs) and Cis-2-decenoic acid inhibit the growth of S. aureus which are discussed below.

\section{Respiratory Toxins}

Among the various virulence factors secreted by $P$. aeruginosa the small respiratory inhibitors such as hydrogen cyanide $(\mathrm{HCN})$, pyocyanin and quinoline $\mathrm{N}$-oxides are the most well studied; and have been found to impact greatly the interactions between the two bacteria. These factors target the electron transport chain (ETC) and suppress the growth of $S$. aureus and also modulate other competing bacterial species.

P. aeruginosa produces HCN by the oxidative decarboxylation of glycine by the enzyme HCN synthase that is encoded by the $h c n A B C$ genes (Blumer and Haas, 2000).Transcription of the hon genes was found to be controlled by the quorum sensor regulators LasR and RhlR, global activator GacA, transcriptional regulator, AlgR as well as the anaerobic regulator ANR (Pessi and Haas, 2000; Pessi and Haas, 2001). The production of HCN was proven to be clinically significant in the CF lungs. Cyanide could be detected in the sputum and bronchoalveolar lavage (BAL) fluids obtained from the $P$. aeruginosa-infected CF patients, which also correlated directly with a worsening prognosis (Carroll et al., 2005; Enderby et al., 2009). P. aeruginosa isolates from $\mathrm{CF}$ patients produced higher levels of HCN compared to the laboratory strains. HCN may aid $P$. aeruginosa in eliminating other lung pathogens by blocking their respiratory chain leading to it being the dominant bacterium. The terminal cytochrome oxidase is the cellular target of HCN (Jones and Poole, 1985). The presence of a cyanide insensitive terminal oxidase encoded by $c i o A B$ helps $P$. aeruginosa avoid the toxic effects of cyanide produced by it. This terminal oxidase is closely related to the cytochrome $b d$ quinol oxidases (Cunningham et al., 1997). HCN produced by $P$. aeruginosa blocks the transfer of electrons from the two terminal oxidases, cytochrome $o$ or cytochrome aa 3 to oxygen in the ETC of $S$. aureus and of the other staphylococcal species and inhibits their aerobic respiration (Voggu et al., 2006).

$P$. aeruginosa synthesizes copious amounts of redox active pigmented secondary metabolite, pyocyanin (PYO), whose production is regulated by $\mathrm{PQS}$ and $\mathrm{Rhl}$ quorum-sensing systems (Welsh et al., 2015). PYO acts against other commensal microbiota and is toxic to a variety of host cells. Its toxicity is attributable to the inhibition of aerobic respiration and to the generation of reactive oxygen species (ROS) such as hydrogen peroxide $\left(\mathrm{H}_{2} \mathrm{O}_{2}\right)$ and superoxide radical $\left(\mathrm{O}_{2}^{-}\right)$ (Hassan and Fridovich, 1980; Noto et al., 2017). PYO was found in the sputum samples obtained from the lungs of CF patients in concentrations as high as $16 \mu \mathrm{g} / \mathrm{ml}$ which also correlates with the progression of the disease (Wilson et al., 1988). PYO targets the $S$. aureus ETC and interferes with the transfer of electrons and diverts them to produce ROS that can induce death (Hassan and Fridovich, 1980). P. aeruginosa escapes the effects of PYO and its ROS by increasing the activities of the superoxide dismutase (SOD) and catalase under pyocyanin-producing conditions (Hassett et al., 1992). It is speculated that PYO inhibits the transfer of electrons either from the menaquinone pool to cytochrome $b$ or from the two terminal oxidases to oxygen or it acts at both these sites (Voggu et al., 2006). Selection of S. aureus on PYO yielded menadione 
auxotrophic SCVs that were capable of growth at high concentrations of PYO (Noto et al., 2017).

$P$. aeruginosa produces a mixture of low molecular weight, hydrophobic alkyl-hydroxyquinoline $\mathrm{N}$-oxides (AQNOs) that vary in the length and degree of saturation of the alkyl chains. Examples of AQNQs are 2-alkyl-4-quinolone N-oxides, 2heptyl-4-quinolone $\mathrm{N}$-oxide (HQNO) and trans- $\Delta(1)$-2-(non1-enyl)-4-quinolone $\mathrm{N}$-oxide. The AQNOs block the oxidation of cytochrome $b$ and the reduction of cytochrome $c$ and in $S$. aureus they block the oxidation of cytochrome $b 1$ and reduction of cytochrome a2 (Kogut and Lightbown, 1962; Machan et al., 1992). Several studies have demonstrated that at higher concentrations AQNOs inhibit growth and at lower concentrations they induced the selection of SCVs in S. aureus (Szamosvari and Böttcher, 2017).

\section{Staphylolysin (LasA)}

$P$. aeruginosa secretes a $20-\mathrm{kDa}$ metalloendopeptidase, LasA or staphylolysin that cleaves the glycyl-alanine and glycyl-glycine bonds of the pentaglycine crosslink in the peptidoglycan of $S$. aureus and induces its lysis (Lache et al., 1969; Kessler et al., 1993). P. aeruginosa synthesizes small, high-affinity ironchelating compounds siderophores, pyoverdine, and pyochelin (Hoegy et al., 2014). It can also acquire iron via the uptake of heme molecules from the host hemoproteins. Mashburn et al. have found that $S$. aureus can be lysed by $P$. aeruginosa and that the lysed $S$. aureus cells could serve as a source of iron for $P$. aeruginosa. The gene expression patterns of the iron-regulated genes of $P$. aeruginosa were found to be similar when it is grown in the presence of $S$. aureus or in high-iron conditions indicating that $S$. aureus might serve as a source of iron for P. aeruginosa (Mashburn et al., 2005).

\section{Rhamnolipids}

$P$. aeruginosa produces extracellular secondary metabolites called rhamnolipids. These are glycolipids consisting of a dimer of 3-hydroxy fatty acids that are linked via glycosidic bonds to one or two (L)-rhamnose molecules. The stability of the $P$. aeruginosa's biofilm structure relies on the endogenous production of the suitable concentrations of rhamnolipids at the right time. At low concentration they increase the hydrophobicity at the cell surface and enhance aggregation of the $P$. aeruginosa cells and facilitate microcolony formation. Increased hydrophobicity also aids in augmenting the affinity of the cells initial adherence to surfaces. However, at high concentrations they decrease the surface tension and exert an anti-adhesive effect (Pamp and Tolker-Nielsen, 2007). These rhamnolipids have also been found to display anti-adhesive and biofilm dispersal activities that inhibit biofilm formation and disrupt established biofilms formed by several other bacterial and fungal species including $S$. aureus in a dose dependent manner (Wood et al., 2018).

\section{Long Chain N-Acyl Homoserine Lactones (AHLs)}

The long chain AHL, 3-oxo- $\mathrm{C}_{12}$-HSL inhibits the growth of $S$. aureus in a dose dependent manner. At sub-growth inhibitory concentrations, they inhibit the expression of the staphylococcal accessory regulator (sarA), accessory gene regulator (agr) leading to the downregulation of exotoxins (hemolysins, TSST-1) production in S. aureus. Inhibition of SarA leads to enhanced expression of protein A (Qazi et al., 2006). Inhibition of agr in $S$. aureus by the long chain AHLs of $P$. aeruginosa results in enhanced production of surface adhesins such as fibronectin binding proteins $(\mathrm{FnbAB})$ and clumping factor $\mathrm{B}(\mathrm{ClfB})$ that reduce detachment and augment robust biofilm formation and enhance host cell invasion of S. aureus (Schmidt et al., 2003; Qazi et al., 2006; Tamber and Cheung, 2009) (Figure 2).

\section{Cis-2-Decenoic Acid}

Cis-2-decenoic acid (cis-DA), a small messenger molecule produced by $P$. aeruginosa that induces biofilm dispersal in a broad range of bacteria (including $P$. aeruginosa itself and $S$. aureus) and in yeast. It also has been found to enhance metabolic activity, reverse persistence, and increase the activity of antimicrobial agents. The reversal of persister cells to the antimicrobial-susceptible state by cis-DA has been attributed to the increased membrane permeability which allows increased uptake of antimicrobials (Marques et al., 2014). Cis-DA of $P$. aeruginosa makes $S$. aureus susceptible towards host defense system and antimicrobial agents.

\section{S. aureus STRATEGIES TO EVADE KILLING BY $P$. aeruginosa}

It has been observed that as the patients' age, the prevalence of $S$. aureus decreases and $P$. aeruginosa becomes the major pathogen in the adults. Most of the interaction studies have reported that during early co-infections $P$. aeruginosa outcompetes $S$. aureus by employing several strategies that range from secreting virulence products that inhibit the growth or lysis $S$. aureus. However, patients harboring both $S$. aureus and $P$. aeruginosa are detected frequently in the worldwide cohort indicating that $S$. aureus is able to survive and persist in the presence of $P$. aeruginosa. The isolation of $S$. aureus and $P$. aeruginosa from the same lobe of CF lungs suggests that they share the same ecological niche and interact in vivo (Hubert et al., 2013; Baldan et al., 2014). Co-existence of $S$. aureus and $P$. aeruginosa is associated with chronic infection and a faster decline in the lung function (Briaud et al., 2020). To evade P. aeruginosa mediated killing $S$. aureus forms SCVs and up-regulate expression of genes involved in metabolism, which we have discussed below.

\section{Formation of 'Small Colony Variants' (SCV) by $S$. aureus Evades $P$. aeruginosa Mediated Killing}

Despite the presence of the respiratory inhibitors (HCN, PYO and quinoline $N$-oxides) produced by $P$. aeruginosa that block the transfer of electrons in the ETC and suppress its growth, $S$. aureus survives the respiratory attack and sustains infection by adapting to respiration-defective small colony variant (SCV) phenotype (Hoffman et al., 2006; Biswas et al., 2009). These 


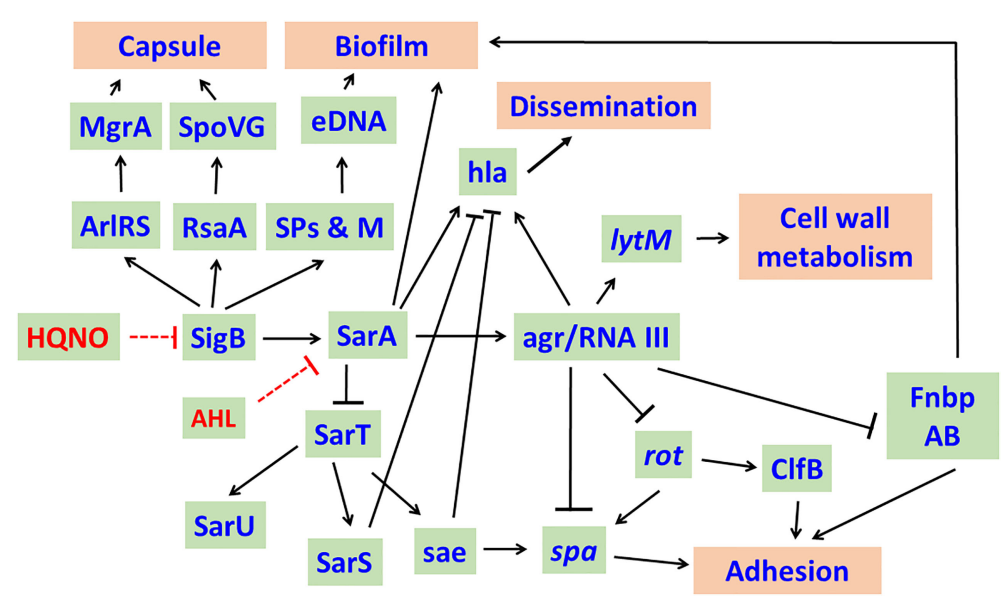

FIGURE 2 | Effect of Long chain - acyl homo serine lactones (AHLs) of $P$. aeruginosa on S. aureus. In general, SigB and SarA regulate the expression of a number of genes and their products and enhance the virulence of $S$. aureus. AHLs of $P$. aeruginosa target SigB and SarA and cause decreased transcription of sigB, sarA and agr RNAlll resulting in decreased capsule production, biofilm formation and dissemination. Inhibition of agr RNAlll enhances the production of Protein A, ClfB, FnbpAB, which increase the adhesion of $S$. aureus to host cells. RpiRc regulates RsbU to modulate eDNA-dependent biofilm formation and in vivo virulence of $S$. aureus in a mouse model of catheter infection. The network was drawn based on the knowledge acquired from literature (Schmidt et al., 2003; Tamber and Cheung, 2009; Fechter et al., 2014; Andrey et al., 2015). Arrows represent activation while bars represent repression. SPs \& M, Secreted proteins \& metabolites; eDNA, extracellular DNA; ClfB, clumping factor B; FnbpAB, Fibronectin binding proteins A and B; spa, Protein A; hla, alpha-toxin; sae, S. aureus exoproteins.

SCVs cause persistent and recurrent infections and are also resistant to antibiotics such as aminoglycosides, antifolate antibiotics, and to host antimicrobial peptides such as HBD2 and HBD3, LL-37 and lactoferricin B (Figure 3). SCVs also exhibit enhanced host cell invasion and intracellular survival, robust biofilm formation, and increased capsule production through a combination of enhanced SigB activity and decreased Agr activity that results in overexpression of surface proteins and insufficient cytolysin production (Moisan et al., 2006; Mitchell et al., 2010). SCVs of S. aureus grow as tiny, nonhemolytic, non-pigmented colonies and are usually auxotrophic to thymidine, menadione or hemin, or can be of transient SCV phenotype that can revert back to the wild-type phenotype in the absence of the selection pressure (Besier et al., 2007). Thymidine auxotrophic S. aureus SCVs were found to be induced by longterm Trimethoprim-Sulfamethoxazole (SXT) treatment and have increased fitness during SXT Challenge. These SCVs fail to synthesize DNA due to mutations in the thymidylate synthase gene (Kriegeskorte et al., 2015).

Genome sequencing of the menadione auxotrophic S. aureus SCV that grew in the presence of high concentrations of pyocyanin led to the identification of mutations in four genes, $q s r R$, saeS, menD, and NWMN_0006 (Noto et al., 2017). The $q s r R$ gene encodes for a quinone-sensing and response repressor of quinone detoxification genes whose inactivation results in the over-expression of quinone/ROS detoxification enzymes and significant pyocyanin resistance (Ji et al., 2013). The saeS gene encodes for the sensor histidine kinase SaeS which is part of the SaeRS two-component system (TCS) that comprises of SaeS along with the response regulator SaeR. The TCS SaeRS plays a vital role in controlling the production of more than 20 virulence factors including surface proteins, proteases, hemolysins, leukocidins and superantigens (Schäfer et al., 2009). The menD gene encodes for the enzyme 2-succinyl-5-enolpyruvyl-6-hydroxy3-cyclohexene-1-carboxylate synthase [NWMN_0913]), which is necessary for menaquinone biosynthesis.

In addition to the blocking of the electron transport chain, PYO also exerts its toxicity through the generation of ROS. $S$. aureus in general uses superoxide dismutases (SodA/M), catalase (KatA), and the pigment staphyloxanthin to combat ROS such as $\mathrm{O}_{2}^{-}$and $\mathrm{H}_{2} \mathrm{O}_{2}$. However, the production of staphyloxanthin by the $S$. aureus SCVs is significantly reduced and also heme auxotrophs are deficient in catalase. Interestingly, exposure of wild-type $S$. aureus to $\mathrm{H}_{2} \mathrm{O}_{2}$ consistently led to the generation of menadione auxotrophs. The appearance of SCVs from the $\mathrm{H}_{2} \mathrm{O}_{2}$ exposure involved the SOS response that includes DNA doublestrand break repair pathway proteins RexAB, polymerase $\mathrm{V}$, and recombinase A (Painter et al., 2015). Clinical menadioneauxotrophic SCV isolates demonstrated increased $\mathrm{H}_{2} \mathrm{O}_{2}$ resistance with elevated catalase activity, relative to a wild-type revertant. Also, the redox-sensing regulator, Rex aids the respiration deficient $S$. aureus SCVs in avoiding the redox stress by shifting to fermentative metabolism (Pagels et al., 2010; Filkins et al., 2015). The fermentative metabolism also imparts resistance to SCVs against ROS mediated killing by decreasing the need for metabolic enzymes and cytochromes that require iron as a cofactor. Metabolic analysis of the $S$. aureus SCVs has revealed significant increase in the production of lactate, the primary source of energy generation in the fermentative metabolism. P. aeruginosa was found to preferentially consume the $S$. aureus-produced lactate as a carbon source over the medium-supplied glucose (Figure 3). 


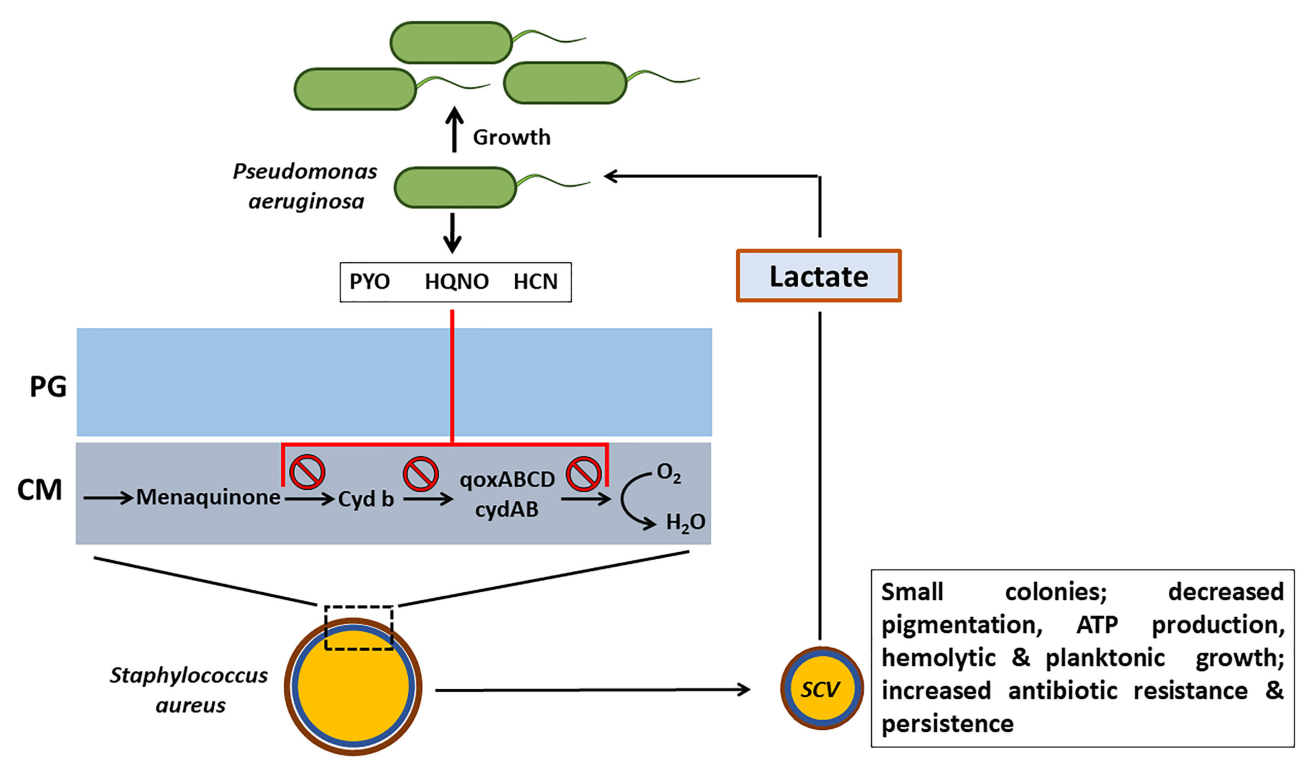

FIGURE 3 | Effect of respiratory inhibitors of $P$. aeruginosa on S. aureus. P. aeruginosa secretes small respiratory inhibitors such as pyocyanin (PYO), hydrogen cyanide $(\mathrm{HCN})$ and quinoline $\mathrm{N}$-oxides (HQNO) that target the electron transport pathway $(\mathrm{ETC})$ and block the transport of electrons. $S$. aureus shifts to fermentative metabolism and adapts the small colony variant (SCV) phenotype to survive. In addition to resisting the respiratory inhibitors of $P$. aeruginosa, SCV phenotype imparts several advantages to $S$. aureus such as increased antibiotic resistance and persistence. The lactate that is produced by $S$. aureus SCVs will be consumed by $P$. aeruginosa as a preferential carbon source. PG, peptidoglycan; $\mathrm{CM}$, cell memebrane.

\section{L-Form-Like S. aureus Cells Evade LasA Mediated Lysis by $P$. aeruginosa}

$S$. aureus survives the activity of LasA produced by $P$. aeruginosa by forming the cell wall deficient L-form-like colonies (Falcon et al., 1989). S. aureus as L-forms were found to internalize, replicate and persist in the lungs of the infected rats by preventing the formation of phagolysomes and digestive vacuoles (Schmitt-Slomska et al., 1986; Michailova et al., 2007). Intracellular persistence as L-forms would provide S. aureus with an opportunity to not only evade professional phagocytes but also protect it from extracellular antibiotics and would promote recrudescent infection (Figure 4).

\section{S. aureus Alters Expression of Metabolic Genes to Encounter Killing by $P$. aeruginosa}

In competitive interactions the major metabolic pathways such as Krebs cycle (downregulation of Acetyl-coA synthetase resulting in reduced ATP production), translation (increased levels of tRNAs and ribosomal RNAs are associated with reduced translation efficiency) and oxidative stress of $S$. aureus were found to be affected. Upregulation of several dehydrogenase enzymes, such as lactate dehydrogenase $(\operatorname{ldh} A)$, glutamate dehydrogenase $(g l u D)$, alanine dehydrogenase (ald 1$), 2$ oxoglutarate dehydrogenase (odhA), 1-pyrroline-5-carboxylate dehydrogenase $(\operatorname{roc} A)$ and aldehyde-alcohol dehydrogenase $(a d h E)$ as well as the L-lactate permease $(l c t P)$ suggests a switch to lactic acid fermentation from aerobic respiration. $P$. aeruginosa consumes the lactate generated by $S$. aureus as a carbon source. The $a d h E$ and gluD are also associated with oxidative stress responses (Briaud et al., 2019). Additionally, the genes $p g i$ (glucose-6-phosphate isomerase), $f b p$ (fructosebisphosphate aldolase) and $f d a$ (fructose-1,6-bisphosphatase) that were involved in glycolysis and pentose phosphate pathways were down-regulated indicating a competition for nutrients and that $S$. aureus uses sources other than glucose to produce energy and nucleotides.

The presence of $P$. aeruginosa also effects the expression of the genes involved in the nucleotide metabolism in $S$. aureus. The two $n r d$ operons ( $n r d E, n r d I, n r d F$ and $n r d G, n r d D$ ) that regulate the production of deoxyribonucleotide di- or triphosphates were down-regulated while the $d e o D$ gene that encodes for purine nucleoside phosphorylase which plays a major role in alternative metabolic pathway for nucleotides was upregulated (Briaud et al., 2019). As the $n r d$ operons play a vital role in DNA synthesis and its repair control, limiting the synthesis of deoxyribonucleotide phosphates will affect the cell concentration.

In response to the co-culture induced nitrogen source limitation particularly purines, urea, and amino acids (mostly glutamine), $P$. aeruginosa up-regulated its master nitrogen regulator gene, $n \operatorname{tr} C$ and other genes involved in nitrogen assimilation such as $g d h A$ (glutamate dehydrogenase), $g \ln A$ (glutamine synthase) and ureE (urease accessory factor). Also the expression of the glutamine amido/aminotransferase encoding operon PA14_06890-PA14_06930 operon was enhanced in the presence of $S$. aureus. While in response to nitrogen starvation $S$. aureus reduced the expression of its glutamate synthase encoding genes, gltB and gltD that convert glutamine to glutamate. Moreover, increased expression of glutamine binding protein (NWMN_1750) in $S$. aureus and ammonium transporter (PA14_24780) in $P$. 


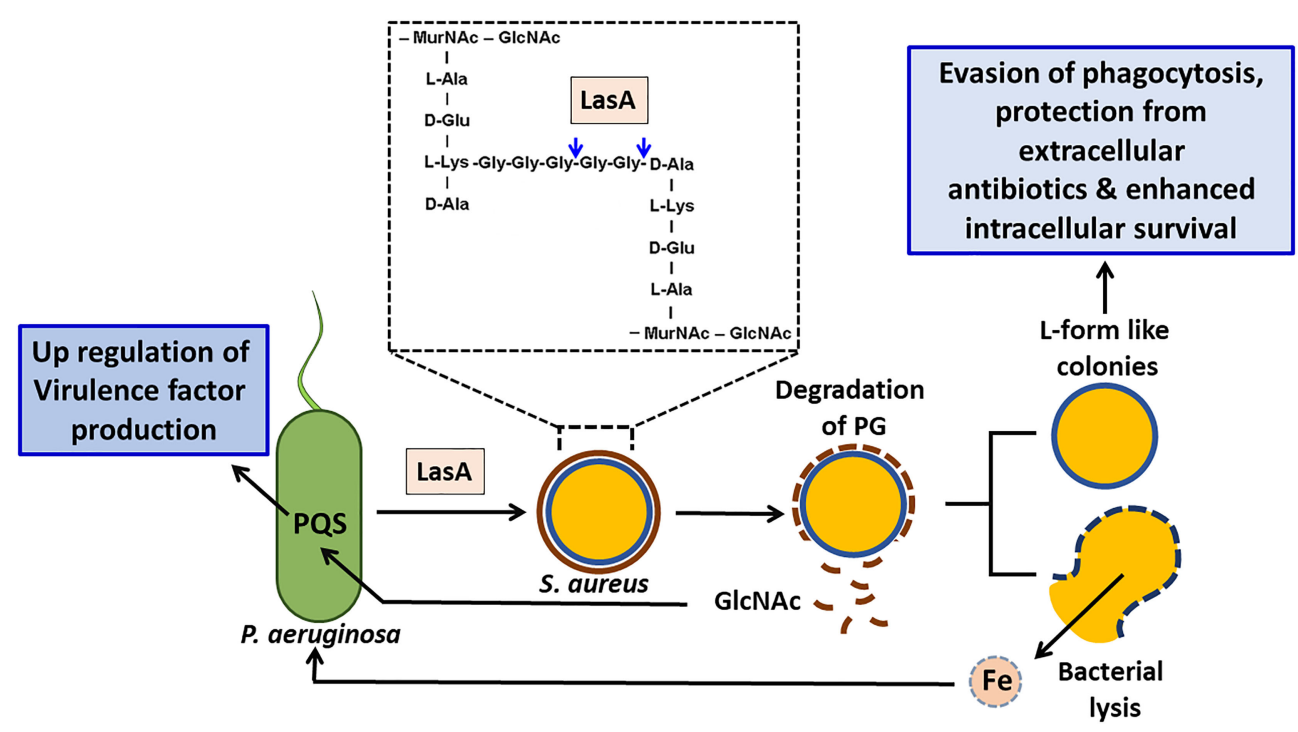

FIGURE 4 | Effect of staphylolysin (LasA) of $P$. aeruginosa on S. aureus. P. aeruginosa secreted staphylolysin (LasA) targets the glycyl-glycine and glycyl-alanine bonds of the pentaglycine cross-linkage in the peptidoglycan of $S$. aureus and induces its lysis. Lysed $S$. aureus cells could serve as a source of iron for $P$. aeruginosa thus promotes its growth. The $N$-acetyl glucosamine (GlcNAc) that is shed will be sensed by $P$. aeruginosa and enhances its PQS quorum sensing system and induces the production of extracellular virulence factors such as PYO, rhamnolipids and LasA etc that will aid in eliminating its competitors. S. aureus adapts the L-form like colonies to survive. In addition to surviving the effects of LasA, L-form like colonies confer several advantages to $S$. aureus such as evasion of phagocytosis, enhanced intracellular survival and protection from the effect of extracellular antibiotics.

aeruginosa indicate that both bacteria try to acquire nitrogen from extracellular sources. These modifications suggest that coexistence induces competition for nutrient resources and triggers global responses that are mostly dominated by metabolic adaptations (Tognon et al., 2019). In response to the stress caused due to nutrient limitation or cell damage both these pathogens were found to trigger lysogenic mechanisms as was evidenced by the induction of the prophages in S. aureus and Rand $\mathrm{F}$ - pyocin synthesis genes in $P$. aeruginosa (Tognon et al., 2019). R-type, S-type, and F-type pyocins are bacteriocins that are secreted by $P$. aeruginosa into the environment to reduce competition from other bacterial strains. They differ in their morphology and mode of killing (Nakayama et al., 2000). The cell lysis resulting from the induction of pyocin and prophages in a small sub-population of the culture could aid in sensing the presence of a resource competitor. $P$. aeruginosa also has the ability to sense the peptidoglycan component $\mathrm{N}$ acetylglucosamine (GlcNAc) that is shed by the Gram-positive commensal flora during cell lysis or cell wall turnover. Community surveillance by sensing GlcNAc aids $P$. aeruginosa in eliminating its competitors by enhancing the PQS QS induced production of virulence factors such as PYO, rhamnolipids and LasA etc (Korgaonkar et al., 2013).

\section{In Coexistence With S. aureus, $P$. aeruginosa Loses Virulence}

During co-existence with $P$. aeruginosa, $S$. aureus employs several strategies to survive the attack of $P$. aeruginosa such as formation of SCVs and altering the expression patterns of several of its genes. Recently it was found that $S$. aureus also influences the overall virulence of $P$. aeruginosa. The strains of $P$. aeruginosa coexisting with $S$. aureus were found to be less aggressive compared to the strains isolated from the monoinfected patients which were more competitive towards $S$. aureus and exhibited antagonistic interactions. It was observed that the early and late clonal variants of $P$. aeruginosa adopt different phenotypes that influence the growth of $S$. aureus differently. While the early-infecting $P$. aeruginosa strains had a negative effect on $S$. aureus growth and had competitive interactions, $P$. aeruginosa strains in the later stages of chronic infection exhibited significantly attenuated virulence towards $S$. aureus. During chronic infections in the process of adapting to the CF lungs, $P$. aeruginosa undergoes microevolution that results in the acquisition of mucoidy, enhanced antibiotic resistance, loss of motility, and loss-of function mutations in virulence genes (Baldan et al., 2014). P. aeruginosa isolates with the mucoid phenotype were found to be the least competitive among the co-isolates. Acquisition of the mucoid phenotype results from the loss-of-function mutation within the mucA gene that encodes for an anti-sigma factor that usually prevents the synthesis of alginate, a polymer of $\mathrm{O}$-acetylated $\mathrm{D}$-mannuronic acid and L-guluronic acids. The loss-of-function mutation within the $m u c A$ gene was found to downregulate the transcriptionof the gene $p v d A$ that leads to reduced production of a subset of virulence factors such as rhamnolipids, pyoverdine, and 2heptyl-4-hydroxyquinolone- $N$-oxide (HQNO) that act against $S$. aureus. Exogenous alginate thereby prevents the killing of $S$. aureus and promotes the coexistence of $P$. aeruginosa with $S$. aureus within the CF lung (Limoli et al., 2017; Price et al., 2020). Interestingly, exogenous alginate was found to protect both 
planktonic cells and biofilms of $S$. aureus from antibiotics suggesting a shift from competitive to cooperative interactions between the two pathogens (Orazi and O'Toole, 2017).

\section{CO-OPERATIVE INTERACTIONS BETWEEN S. AUREUS AND $P$. aeruginosa}

In addition to these competitive interactions several co-operative interactions between $S$. aureus and $P$. aeruginosa have also been reported. When $S$. aureus was co-cultured with $P$. aeruginosa it was found to induce mutations in the genes encoding the lipopolysaccharide (LPS) biosynthesis which confers fitness gain and is also associated with enhanced resistance towards $\beta$ lactam antibiotics (Tognon et al., 2017). Exoproducts of S. aureus were also found to restore and enhance swimming and swarming motility of $P$. aeruginosa in an isolate dependent manner (Pallett et al., 2019). Protein A produced by S. aureus exists in both secreted and membrane-bound forms. The secreted Protein A interacts with two specific structures on the cell surface of $P$. aeruginosa, the Psl (Pseudomonas polysaccharide locus) polysaccharide and the PilA protein component of type IV pili. In the absence of Psl, Protein A binds to type IV pili and inhibits biofilm formation by $P$. aeruginosa strains. Protein A binds the $\mathrm{Fc} \gamma$ domain of the immunoglobulin and prevents Fc receptor mediated opsonophagocytosis and killing of S. aureus (Figure 5). It is found to impart similar protection to $P$. aeruginosa from IgG-mediated neutrophil opsonophagocytosis in vitro (Armbruster et al., 2016). HQNO and PQS molecules secreted by $P$. aeruginosa were found to enhance biofilm formation by $S$. aureus (Fugere et al., 2014).

\section{INFLUENCE OF HOST FACTORS ON COEXISTENCE OF S. AUREUS AND $P$. aeruginosa}

S. aureus is the main colonizing bacteria of the CF lungs during infancy and early childhood, its incidence declines thereafter and infections by $P$. aeruginosa become more prominent with increasing age. Reduced mucociliary clearance, over-sulfation of the glycocalyx, reduced sialylation of apical proteins and increased concentration of asialoganglioside 1 (aGM1) in apical membranes of CF epithelia likely aid in the adherence and subsequent colonization of $S$. aureus and P. aeruginosa and contribute to the pathogenesis of these bacterial infections in the CF lung (Cheng et al., 1989; Michailova et al., 2007). Altered ionic composition, volume and $\mathrm{pH}$ of the airway surface liquid (ASL) due to defective CFTR function play critical roles in the pathophysiology of CF (Verkman et al., 2003). Abnormally low $\mathrm{pH}$ of the ASL in the CF bronchial epithelial cells was found to play a significant role in the initial bacterial colonization of the $S$. aureus within the first hours of life. Defective cAMP-dependent bicarbonate secretion that involves CFTR and SLC26A4 (pendrin) and persistent $\mathrm{H}^{+}$secretion by ATP12A have been found to play a role in the abnormal acidification of the ASL. The major antimicrobial peptides (AMPs) in the lung tissues and secretions are cathelicidin LL-37, neutrophil $\alpha$-defensins/ Human Neutrophil Peptides, HBDs, Secreted group IIA phospholipase A2 (sPLA2-IIA) (Hiemstra et al., 2016). In the healthy lungs, these AMPs play a critical role in protection against infection by pathogens. In CF lungs, the $\mathrm{pH}$ of the ASL compromises the activity of the AMPs such as LL-37 and HBD1 which is vital for $S$. aureus clearance from the airways (Simonin et al., 2019). The abnormally high $\mathrm{NaCl}$ concentration in the $\mathrm{CF}$ ASL also interferes with the activity of AMPs such as HBD-1 (Goldman et al., 1997). The predominance of S. aureus in lung expectorate early in life of the CF patients could be related to the CF-specific defective immune response that is dependent on the $\mathrm{pH}$ and ionic composition of the CF ASL. P. aeruginosa has been reported to manipulate the host immune response by inducing strong expression of the cationic enzyme, sPLA2-IIA via a Krüppel-like factor 2 (KLF2) transcription factor dependent pathway. Of the four type-III secretion system (T3SS) effector molecules (ExoS, ExoT, ExoU and ExoY) produced by $P$. aeruginosa, ExoS, has been found to be involved in the induction of the expression of sPLA2-IIA by the host cells (Belmadi et al., 2018). The levels of the sPLA2-IIA produced are adequate to kill $S$. aureus with little effect on $P$. aeruginosa (Pernet et al., 2014). This could be a possible contributing factor for the shift in the infections from $S$. aureus in the early stages of CF to $P$. aeruginosa as the major pathogen in the later stages.

Anoxia arising due to static mucus, reduced pulmonary function and consumption of oxygen by host neutrophils facilitates co-existence of $S$. aureus and $P$. aeruginosa in the CF lung. Under anoxia most $P$. aeruginosa $\mathrm{CF}$ isolates were unable to antagonize the growth of S. aureus (Pallett et al., 2019). Physiological concentration of serum albumin was shown to promote coexistence of $S$. aureus and $P$. aeruginosa by inhibiting quorum sensing in $P$. aeruginosa by sequestering its AHLs and repressing the production of virulence factors that can kill $S$. aureus (Smith et al., 2017). Additionally, the host innate immune protein calprotectin was shown to promote co-existence of $S$. aureus and $P$. aeruginosa by sequestering zinc- and manganese metal ions and repressing the production of anti-staphylococcal compounds by $P$. aeruginosa (Wakeman et al., 2016). Volatile molecules present in the BAL fluid might aid in ruling in $P$. aeruginosa and ruling out $S$. aureus infections in CF patients (Nasir et al., 2018).

\section{THE INTERACTIONS OF S. aureus AND $P$. aeruginosa LEADS TO INCREASED ANTIMICROBIAL RESISTANCE}

Interspecies interactions within the microbial community in the $\mathrm{CF}$ lungs influence the efficacy of different antibiotics. Both $P$. aeruginosa and $S$. aureus have the ability to form biofilms and exhibit intrinsic and acquired resistance to antibiotics which makes treating their infections difficult. In the CF lungs both these 


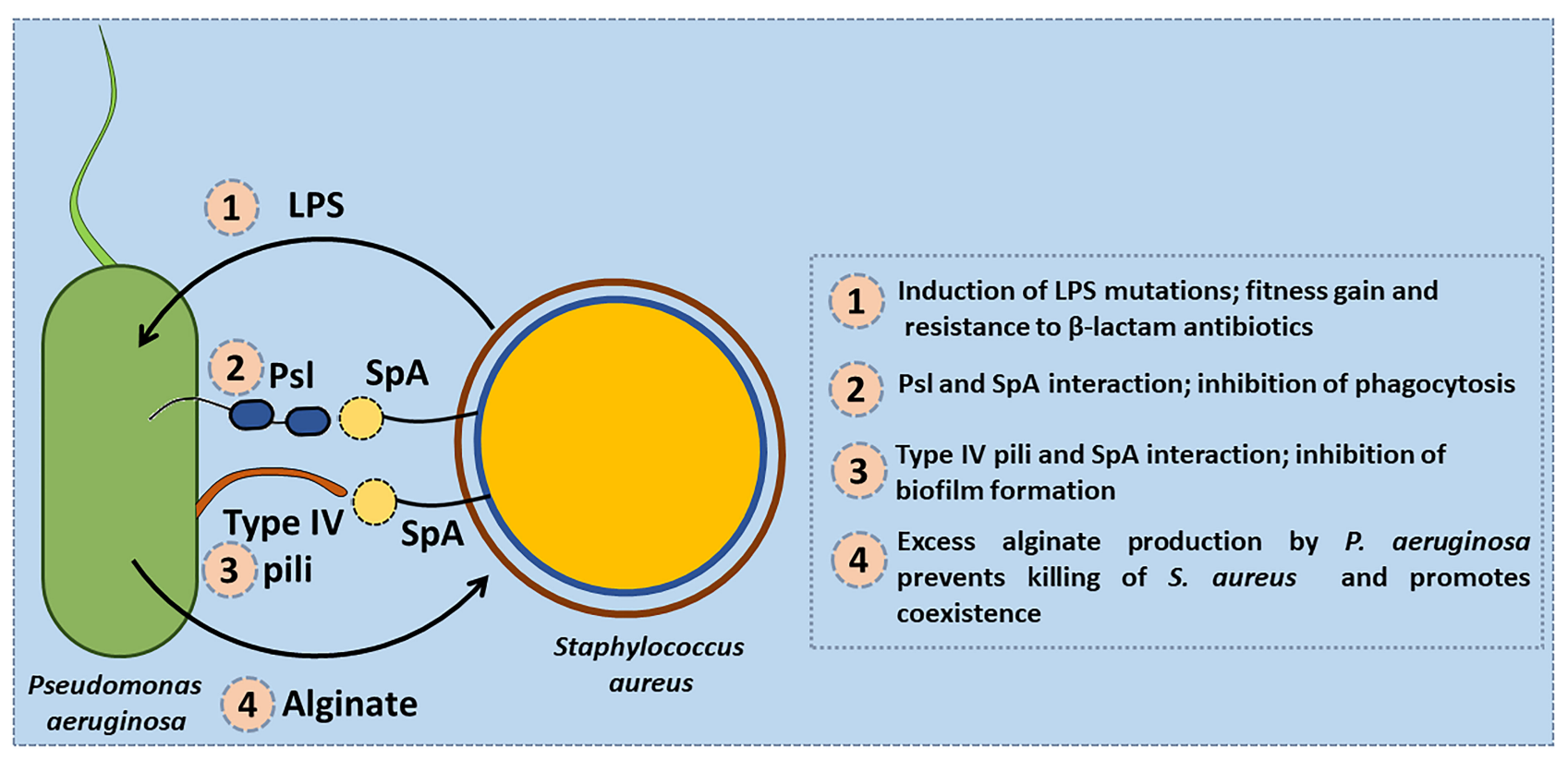

FIGURE 5 | Co-operative interactions between S. aureus and P. aeruginosa. (1) S. aureus induces mutations in the genes encoding the lipopolysaccharide (LPS) biosynthesis in $P$. aeruginosa that confers fitness gain and enhanced resistance to $\beta$-lactam antibiotics. (2) Protein A (SpA) produced by $S$. aureus interacts with the Psl (Pseudomonas polysaccharide locus) polysaccharide of $P$. aeruginosa and protects it from being phagocytosed by neutrophils. (3) SpA interacts with type IV pili of $P$. aeruginosa and inhibits biofilm formation by $P$. aeruginosa strains. (4) Exogenous alginate secreted by $P$. aeruginosa protects $S$. aureus by reducing the production of virulence factors such as siderophores, and rhamnolipids.

pathogens adapt a biofilm-like growth as the hypoxia and the thick, dehydrated mucus provide optimal conditions for biofilm formation. Encased within their extracellular polysaccharide matrices these pathogens exhibit 100-1000-fold more resistance to antibiotics and host immune responses compared to their planktonic counterparts because of poor penetration and slow growth (SCV formation). P. aeruginosa secreted HQNO, PYO and HCN block the ETC in the $S$. aureus which in turn resorts to SCV formation by shifting to fermentative metabolism resulting in decreased ATP production (Hoffman et al., 2006; Voggu et al., 2006; Biswas et al., 2009; Götz and Mayer, 2013). Reduced ATP negatively impacts the active transport which is essential for the a minoglycoside antibiotics to gain entry into the cells. These SCVs display enhanced tolerance to several antimicrobial agents (Lechner et al., 2012). Similarly, the presence of S. aureus was also found to promote the selection of SCVs in P. aeruginosa that exhibit increased resistance to several antibiotics (Michelsen et al., 2014). These interactions during coexistence were found to be mediated by the agr quorum sensing system, the global regulator SarA and the ClpP proteolytic complexes which regulate the expression of major virulence factors in $S$. aureus.

The presence of $P$. aeruginosa was found to induce the overexpression of nor genes (tet 38 , nor $A$, and nor C) in $S$. aureus. The efflux pump proteins encoded by these nor genes are responsible for the increased antibiotic resistance against tetracycline and fluoroquinolone (ciprofloxacin). They also promote internalization of $S$. aureus by the host epithelial cells (Briaud et al., 2019). LasA endopeptidase and rhamnolipids produced by
$P$. aeruginosa enhanced the killing of $S$. aureus by vancomycin and tobramycin, by enhancing lysis and facilitating protonmotive force-independent uptake, respectively (Radlinski et al., 2017). In mixed species biofilms, $P$. aeruginosa decreased the susceptibility of $S$. aureus to a broad range of antimicrobials such as tetracycline, vancomycin, ampicillin and ceftriaxone while increasing the sensitivity towards quinolones through the secretion of metabolites such as HQNO and siderophores pyoverdine and pyochelin (Orazi and O'Toole, 2017). S. aureus in mixed cultures was also found to increase the sensitivity of $P$. aeruginosa to ciprofloxacin and aminoglycosides antibiotics by tenfold compared to the monocultures (Trizna et al., 2020). The protein A of $S$. aureus was shown to increase the resistance of $P$. aeruginosa to inhaled tobramycin. In the presence of $S$. aureus, $P$. aeruginosa synthesizes truncated LPS that lack O-specific antigen (OSA) which imparts more resistance to $\beta$-lactam antibiotics but not against ciprofloxacin or polymyxin (Tognon et al., 2017). These studies indicate that in mixed biofilms $P$. aeruginosa acquires resistance against antibiotics that target mostly the cell wall and protein biosynthesis but the other resistance mechanisms might remain largely unaffected.

\section{INTERACTION OF P. aeruginosa WITH OTHER STAPHYLOCOCCAL SPECIES}

Earlier studies have shown that $S$. aureus has three different terminal cytochrome oxidases, the heme/copper oxidases 
[cytochrome aa3 (QoxABCD) and cytochrome $o$ ] and the hemeonly cytochrome $b d$ quinol oxidase (CydAB) (Faller et al., 1980; Voggu et al., 2006; Götz and Mayer, 2013; Hammer et al., 2016).The respiratory inhibitors secreted by $P$. aeruginosa exert their toxic effects not only on $S$. aureus but also on the other pathogenic staphylococcal species including S. epidermidis, $S$. haemolyticus, S. hyicus, S. lugdunensis, S. muscae and S. saprophyticus. Interestingly, the non-pathogenic staphylococcal species such as S. carnosus, S. gallinarum, S. lentus, S. piscifermentans and S. simulans are resistant to these toxins. The resistance of the non-pathogenic staphyloccal species lies in the presence of a functional alternative terminal oxidase, the pyocyanin and cyanide resistant cytochrome $b d$ quinol oxidase, (CydAB). It oxidises ubiquinol and reduces oxygen as part of the ETC and is composed of two subunits, CydA and CydB. While both pathogenic and non-pathogenic staphylococci harbour CydAB terminal oxidase, the sequence of the CydA was found to be more conserved than $\mathrm{CydB}$ among staphylococci (Voggu et al., 2006). The structural alterations in the CydB have been reported to govern the impact of the respiratory inhibitors to block respiration and thereby influence the viability of the pathogenic staphylococci (Voggu et al., 2006). The microevolution of the CydB could possibly be due to the fact that the non-pathogenic staphylococci might have been under pressure for selection of a greater resistance to the respiratory toxins as they frequently inhabit the same ecological niche as Pseudomonas species than the pathogenic staphylococci (Voggu et al., 2006).

Disruption of the QS mediated communication of the $P$. aeruginosa by the quorum quenching enzymes and quorum sensing inhibitors (QSI) could lead to decreased virulence factor production. Staphylococcus intermedius, S. delphini, S. pseudintermedius, S. lutrae and S. schleiferi are the coagulasepositive bacterial species that belong to the Staphylococcus intermedius group (Fitzgerald, 2009; Ben Zakour et al., 2012). They are primarily zoonotic pathogens and rarely cause severe zoonotic infections of humans. These staphylococcal species were also found to secrete the so-called trace amines (TAs) like phenethylamine, tyramine, or tryptamine which boost internalization by host cells and interact with adrenergic receptors (Luqman et al., 2018; Luqman et al., 2019). Urea derivatives of trace amines, like N-[2-(1H-indol-3-yl)ethyl]urea and $\mathrm{N}$-(2-phenethyl)-urea, which were named yayurea $\mathrm{A}$ and $\mathrm{B}$, suppress the virulent factor production in not only $P$. aeruginosa but also in other Gram-negative bacteria such as Vibrio harveyi, Serratia marcescens, and Chromobacterium subtsugae (Chu et al., 2013). While the yayurea A and B do not inhibit growth their QS inhibiting effects suppress the QS dependent virulent factor production including the production of PYO and other respiratory toxins in P. aeruginosa. When yayurea $\mathrm{A}$ and $\mathrm{B}$ were added to the medium $S$. aureus was protected from the growth inhibition by $P$. aeruginosa without the former having to undergo physiological transformation into the SCV (Chu et al., 2013).

Many Gram-negative bacteria employ $N$-acylhomoserine lactone (AHL)-mediated quorum sensing to control virulence.
But surprisingly S. aureus is also responsive to AHLs of Gramnegative bacteria (Qazi et al., 2006). Recently it has been shown that coagulase-negative staphylococci (CNS) such as S. carnosus, S. haemolyticus, S. saprophyticus and S. sciuri secrete a $N$ Acylhomoserine lactonase (AHL-lactonase, ahlS) that is able to degrade $N$-Acylhomoserine lactones in $P$. aeruginosa thus causing a decrease in PYO and elastase production (Morohoshi et al., 2020). This leads us back to our earlier assumption that $S$. aureus is less protected from Pseudomonas, than many CNS species which have PYO and cyanide resistant terminal cytochrome oxidase (Voggu et al., 2006). To survive the respiratory toxins $S$. aureus shifts, or mutates, to SCVs (Biswas et al., 2009). Despite this disadvantage that $S$. aureus has over $P$. aeruginosa, it is amazing how persistently $S$. aureus can colonize lungs of CF patients over a long period of time, alone or together with $P$. aeruginosa. In contrast to many free-living CNS, $S$. aureus may not have learned to defend itself adequately against Pseudomonas. Presumably, S. aureus did not need to do so, since the CF lung is one of the very few habitats in which it encounters $P$. aeruginosa.

\section{DISCUSSION}

Airways of patients with CF are chronically colonized with complex, polymicrobial infections. S. aureus is usually detected at early stages and $P$. aeruginosa becomes predominant at later stages. A number of studies reported antagonistic interactions between these two pathogens. However, clinical data have also documented the co-existence of $P$. aeruginosa and $S$. aureus in CF lungs. In this review we aimed to detail the mechanisms of $P$. aeruginosa and $S$. aureus interactions in the CF environment. $P$. aeruginosa produces a number to toxins that are active against $S$. aureus. However, in the in vivo $\mathrm{CF}$ lung, $P$. aeruginosa may become less aggressive toward $S$. aureus. Overall, three major mechanisms of interaction between $P$. aeruginosa and $S$. aureus were observed: (i) the initial interactions in which $P$. aeruginosa express a number of virulence factors such as PYO, LasA or rhamnolipids to outcompete $S$. aureus, (ii) adaptation of $S$. aureus to SCV and L-forms through phenotypic and genetic modulations to ensure their survival and co-exist in the CF lungs with $P$. aeruginosa, (iii) adaptation of $P$. aeruginosa to the $\mathrm{CF}$ environment alters its QS networks and reduces its virulence factor production and promotes co-existence with $S$. aureus. The modulation of the CF lung environment by $P$. aeruginosa promotes S. aureus colonization. Activation of NF- $\kappa \mathrm{B}$ by $P$. aeruginosa LPS and increased production of TNF- $\alpha$ induced by $P$. aeruginosa was found to increase $S$. aureus invasion in murine lung infection models (Millette et al., 2019). P. aeruginosa infections could also increase the expression of host cell components, ITGA-5 and ICAM-1 which further facilitate $S$. aureus interactions with host epithelial and endothelial cells in the lung environment. Furthermore, S. aureus secreted proteins protect $P$. aeruginosa from opsonophagocytic killing by host. These overall interactions lead to excessive lung damage and 
increased antibiotic resistance in both these pathogens. This review summarized the information available so far regarding $P$. aeruginosa with $S$. aureus interactions in CF lung, however further studied need to be performed for complete understanding of the complex micro-environment present within the CF lung. Although this review discusses the interactions of $P$. aeruginosa with $S$. aureus in CF lung, it is noteworthy to mention that $P$. aeruginosa with $S$. aureus also coexist in the peritoneum of dialysis patients', diabetic foot wounds, catheters, and in the wounds resulting from skin injury or skin burn. The interactions between these pathogens in the extra-pulmonary locations may involve different mechanisms as the host environment would be completely different from the CF lungs and require further investigations.

\section{REFERENCES}

Anderson, M. P., Gregory, R. J., Thompson, S., Souza, D. W., Paul, S., Mulligan, R. C., et al. (1991). Demonstration That Cftr Is a Chloride Channel by Alteration of Its Anion Selectivity. Science 253, 202-205. doi: 10.1126/science.1712984

Andrey, D. O., Jousselin, A., Villanueva, M., Renzoni, A., Monod, A., Barras, C., et al. (2015). Impact of the Regulators SigB, Rot, SarA and sarS on the Toxic Shock Tst Promoter and TSST-1 Expression in Staphylococcus Aureus. PloS One 10, e0135579. doi: 10.1371/journal.pone.0135579

Armbruster, C. R., Wolter, D. J., Mishra, M., Hayden, H. S., Radey, M. C., Merrihew, G., et al. (2016). Staphylococcus Aureus Protein A Mediates Interspecies Interactions at the Cell Surface of Pseudomonas Aeruginosa. Mbio 7, e00538-16. doi: 10.1128/mBio.00538-16

Baldan, R., Cigana, C., Testa, F., Bianconi, I., De Simone, M., Pellin, D., et al. (2014). Adaptation of Pseudomonas Aeruginosa in Cystic Fibrosis Airways Influences Virulence of Staphylococcus Aureus In Vitro and Murine Models of Co-Infection. PloS One 9, e89614. doi: 10.1371/journal.pone.0089614

Belmadi, N., Wu, Y., and Touqui, L. (2018). Immuno-Modulatory Functions of the Type-3 Secretion System and Impacts on the Pulmonary Host Defense: A Role for ExoS of Pseudomonas Aeruginosa in Cystic Fibrosis. Toxicon 143, 68-73. doi: 10.1016/j.toxicon.2018.01.004

Ben Zakour, N. L., Beatson, S. A., Van Den Broek, A. H., Thoday, K. L., and Fitzgerald, J. R. (2012). Comparative Genomics of the Staphylococcus Intermedius Group of Animal Pathogens. Front. Cell Infect. Microbiol. 2, 44. doi: $10.3389 /$ fcimb.2012.00044

Besier, S., Smaczny, C., Von Mallinckrodt, C., Krahl, A., Ackermann, H., Brade, V., et al. (2007). Prevalence and Clinical Significance of Staphylococcus Aureus Small-Colony Variants in Cystic Fibrosis Lung Disease. J. Clin. Microbiol. 45, 168-172. doi: 10.1128/JCM.01510-06

Biswas, L., Biswas, R., Schlag, M., Bertram, R., and Götz, F. (2009). Small-Colony Variant Selection as a Survival Strategy for Staphylococcus Aureus in the Presence of Pseudomonas Aeruginosa. Appl. Environ. Microbiol. 75, 69106912. doi: 10.1128/AEM.01211-09

Blumer, C., and Haas, D. (2000). Mechanism, Regulation, and Ecological Role of Bacterial Cyanide Biosynthesis. Arch. Microbiol. 173, 170-177. doi: 10.1007/ s002039900127

Bobadilla, J. L., Macek, M., Fine, J. P., and Farrell, P. M. (2002). Cystic Fibrosis: A Worldwide Analysis of CFTR Mutations - Correlation With Incidence Data and Application to Screening. Hum. Mutat. 19, 575-606. doi: 10.1002/humu.10041

Briaud, P., Bastien, S., Camus, L., Boyadjian, M., Reix, P., Mainguy, C., et al. (2020). Impact of Coexistence Phenotype Between Staphylococcus Aureus and Pseudomonas Aeruginosa Isolates on Clinical Outcomes Among Cystic Fibrosis Patients. Front. Cell. Infect. Microbiol. 10. doi: 10.3389/ fcimb.2020.00266

Briaud, P., Camus, L., Bastien, S., Doleans-Jordheim, A., Vandenesch, F., and Moreau, K. (2019). Coexistence With Pseudomonas Aeruginosa Alters Staphylococcus Aureus Transcriptome, Antibiotic Resistance and Internalization Into Epithelial Cells. Sci. Rep. 9, 16564. doi: 10.1038/s41598019-52975-z

\section{AUTHOR CONTRIBUTIONS}

LB and FG wrote the manuscript and conceived the figures. LB and FG reviewed the manuscript draft. All authors contributed to the article and approved the submitted version.

\section{FUNDING}

This work was supported by funding from the Deutsche Forschungsgemeinschaft the Germany's Excellence Strategy EXC 2124 - 390838134 'Controlling Microbes to Fight Infections'. We acknowledge support by Open Access Publishing Fund of University of Tübingen, Germany.

Carroll, W., Lenney, W., Wang, T., Spanel, P., Alcock, A., and Smith, D. (2005). Detection of Volatile Compounds Emitted by Pseudomonas Aeruginosa Using Selected Ion Flow Tube Mass Spectrometry. Pediatr. Pulmonol. 39, 452-456. doi: 10.1002/ppul.20170

Cheng, P. W., Boat, T. F., Cranfill, K., Yankaskas, J. R., and Boucher, R. C. (1989). Increased Sulfation of Glycoconjugates by Cultured Nasal Epithelial Cells From Patients With Cystic Fibrosis. J. Clin. Invest. 84, 68-72. doi: 10.1172/ JCI114171

Chu, Y. Y., Nega, M., Wolfle, M., Plener, L., Grond, S., Jung, K., et al. (2013). A New Class of Quorum Quenching Molecules From Staphylococcus Species Affects Communication and Growth of Gram-Negative Bacteria. PloS Pathog. 9, e1003654. doi: 10.1371/journal.ppat.1003654

Clark, S. E. (2020). Commensal Bacteria in the Upper Respiratory Tract Regulate Susceptibility to Infection. Curr. Opin. Immunol. 66, 42-49. doi: 10.1016/ j.coi.2020.03.010

Cunningham, L., Pitt, M., and Williams, H. D. (1997). The cioAB Genes From Pseudomonas Aeruginosa Code for a Novel Cyanide-Insensitive Terminal Oxidase Related to the Cytochrome Bd Quinol Oxidases. Mol. Microbiol. 24, 579-591. doi: 10.1046/j.1365-2958.1997.3561728.x

Cysticfibrosisfoundation (2020). Annual Data Report 2021 (Bethesda, Maryland: Cystic Fibrosis Foundation).

Debraekeleer, M., and Daigneault, J. (1992). Spatial-Distribution of the Df508 Mutation in Cystic-Fibrosis - A Review. Hum. Biol. 64, 167-174.

Deziel, E., Lepine, F., Milot, S., He, J., Mindrinos, M. N., Tompkins, R. G., et al. (2004). Analysis of Pseudomonas Aeruginosa 4-Hydroxy-2-Alkylquinolines (HAQs) Reveals a Role for 4-Hydroxy-2-Heptylquinoline in Cell-to-Cell Communication. Proc. Natl. Acad. Sci. U. S. A. 101, 1339-1344. doi: 10.1073/pnas.0307694100

Enderby, B., Smith, D., Carroll, W., and Lenney, W. (2009). Hydrogen Cyanide as a Biomarker for Pseudomonas Aeruginosa in the Breath of Children With Cystic Fibrosis. Pediatr. Pulmonol. 44, 142-147. doi: 10.1002/ppul.20963

Falcon, M. A., Mansito, T. B., Carnicero, A., and Gutierreznavarro, A. M. (1989). L-Form-Like Colonies of Staphylococcus Aureus Induced by an Extracellular Lytic Enzyme From Pseudomonas Aeruginosa. J. Clin. Microbiol. 27, 16501654. doi: $10.1128 / \mathrm{jcm} \cdot 27.7 .1650-1654.1989$

Faller, A. H., Götz, F., and Schleifer, K. H. (1980). Cytochrome Patterns of Staphylococci and Micrococci and Their Taxonomic Implications. Zentralbl. Bacteriol. Hyg. Abt. 1 Orig. C 1, 26-39.

Fechter, P., Caldelari, I., Lioliou, E., and Romby, P. (2014). Novel Aspects of RNA Regulation in Staphylococcus Aureus. FEBS Lett. 588, 2523-2529. doi: 10.1016/ j.febslet.2014.05.037

Filkins, L. M., Graber, J. A., Olson, D. G., Dolben, E. L., Lynd, L. R., Bhuju, S., et al. (2015). Coculture of Staphylococcus Aureus With Pseudomonas Aeruginosa Drives S-Aureus Towards Fermentative Metabolism and Reduced Viability in a Cystic Fibrosis Model. J. Bacteriol. 197, 2252-2264. doi: 10.1128/JB.00059-15

Fitzgerald, J. R. (2009). The Staphylococcus Intermedius Group of Bacterial Pathogens: Species Re-Classification, Pathogenesis and the Emergence of Meticillin Resistance. Vet. Dermatol. 20, 490-495. doi: 10.1111/j.13653164.2009.00828.x 
Fugere, A., Seguin, D. L., Mitchell, G., Deziel, E., Dekimpe, V., Cantin, A. M., et al. (2014). Interspecific Small Molecule Interactions Between Clinical Isolates of Pseudomonas Aeruginosa and Staphylococcus Aureus From Adult Cystic Fibrosis Patients. PloS One 9, e86705. doi: 10.1371/journal.pone.0086705

Goldman, M. J., Anderson, G. M., Stolzenberg, E. D., Kari, U. P., Zasloff, M., and Wilson, J. M. (1997). Human Beta-Defensin-1 Is a Salt-Sensitive Antibiotic in Lung That is Inactivated in Cystic Fibrosis. Cell 88, 553-560. doi: 10.1016/ S0092-8674(00)81895-4

Götz, F., and Mayer, S. (2013). Both Terminal Oxidases Contribute to Fitness and Virulence During Organ-Specific Staphylococcus Aureus Colonization. Mbio 4, e00976-13. doi: 10.1128/mBio.00976-13

Hammer, N. D., Schurig-Briccio, L. A., Gerdes, S. Y., Gennis, R. B., and Skaar, E. P. (2016). CtaM Is Required for Menaquinol Oxidase Aa3 Function in Staphylococcus Aureus. mBio 7, e00823-16. doi: 10.1128/mBio.00823-16

Hassan, H. M., and Fridovich, I. (1980). Mechanism of the Antibiotic Action Pyocyanine. J. Bacteriol. 141, 156-163. doi: 10.1128/jb.141.1.156-163.1980

Hassett, D. J., Charniga, L., Bean, K., Ohman, D. E., and Cohen, M. S. (1992). Response of Pseudomonas Aeruginosa to Pyocyanin: Mechanisms of Resistance, Antioxidant Defenses, and Demonstration of a ManganeseCofactored Superoxide Dismutase. Infect. Immun. 60, 328-336. doi: 10.1128/ iai.60.2.328-336.1992

Hiemstra, P. S., Amatngalim, G. D., van der Does, A. M., and Taube, C. (2016). Antimicrobial Peptides and Innate Lung Defenses: Role in Infectious and Noninfectious Lung Diseases and Therapeutic Applications. Chest 149, 545551. doi: 10.1378/chest.15-1353

Hoegy, F., Mislin, G. L. A., and Schalk, I. J. (2014). Pyoverdine and Pyochelin Measurements. Pseudomonas: Methods Protoc. 1149, 293-301. doi: 10.1007/ 978-1-4939-0473-0_24

Hoffman, L. R., Deziel, E., D’argenio, D. A., Lepine, F., Emerson, J., Mcnamara, S., et al. (2006). Selection for Staphylococcus Aureus Small-Colony Variants Due to Growth in the Presence of Pseudomonas Aeruginosa. Proc. Natl. Acad. Sci. U. S. A. 103, 19890-19895. doi: 10.1073/pnas.0606756104

Hubert, D., Reglier-Poupet, H., Sermet-Gaudelus, I., Ferroni, A., Le Bourgeois, M., Burgel, P. R., et al. (2013). Association Between Staphylococcus Aureus Alone or Combined With Pseudomonas Aeruginosa and the Clinical Condition of Patients With Cystic Fibrosis. J. Cyst. Fibros. 12, 497-503. doi: 10.1016/j.jcf.2012.12.003

Ji, Q. J., Zhang, L., Jones, M. B., Sun, F., Deng, X., Liang, H. H., et al. (2013). Molecular Mechanism of Quinone Signaling Mediated Through SQuinonization of a YodB Family Repressor QsrR. Proc. Natl. Acad. Sci. U. S. A. 110, 5010-5015. doi: 10.1073/pnas.1219446110

Jones, J. W., and Poole, R. K. (1985). 10 The Analysis of Cytochromes. Methods Microbiol. 18, 285-328. doi: 10.1016/S0580-9517(08)70479-3

Juhas, M., Eberl, L., and Tummler, B. (2005). Quorum Sensing: The Power of Cooperation in the World of Pseudomonas. Environ. Microbiol. 7, 459-471. doi: $10.1111 /$ j.1462-2920.2005.00769.x

Kessler, E., Safrin, M., Olson, J. C., and Ohman, D. E. (1993). Secreted LasA of Pseudomonas Aeruginosa Is a Staphylolytic Protease. J. Biol. Chem. 268, 75037508. doi: 10.1016/S0021-9258(18)53203-8

Kogut, M., and Lightbown, J. W. (1962). Selective Inhibition by 2-Heptyl-4Hydroxyquinoline N-Oxide of Certain Oxidation-Reduction Reactions. Biochem. J. 84, 368-382. doi: 10.1042/bj0840368

Korgaonkar, A., Trivedi, U., Rumbaugh, K. P., and Whiteley, M. (2013). Community Surveillance Enhances Pseudomonas Aeruginosa Virulence During Polymicrobial Infection. Proc. Natl. Acad. Sci. U. S. A. 110, 10591064. doi: 10.1073/pnas.1214550110

Kriegeskorte, A., Lore, N. I., Bragonzi, A., Riva, C., Kelkenberg, M., Becker, K., et al. (2015). Thymidine-Dependent Staphylococcus Aureus Small-Colony Variants Are Induced by Trimethoprim-Sulfamethoxazole (SXT) and Have Increased Fitness During SXT Challenge. Antimicrob. Agents Chemother. 59, 7265-7272. doi: 10.1128/AAC.00742-15

Lache, M., Hearn, W. R., Zyskind, J. W., Tipper, D. J., and Strominger, J. L. (1969). Specificity of a Bacteriolytic Enzyme From Pseudomonas Aeruginosa. J. Bacteriol. 100, 254-25+. doi: 10.1128/jb.100.1.254-259.1969

Lechner, S., Lewis, K., and Bertram, R. (2012). Staphylococcus Aureus Persisters Tolerant to Bactericidal Antibiotics. J. Mol. Microbiol. Biotechnol. 22, 235-244. doi: 10.1159/000342449

Limoli, D. H., Whitfield, G. B., Kitao, T., Ivey, M. L., Davis, M. R.Jr., Grahl, N., et al. (2017). Pseudomonas Aeruginosa Alginate Overproduction Promotes
Coexistence With Staphylococcus Aureus in a Model of Cystic Fibrosis Respiratory Infection. mBio 8, e00186-17. doi: 10.1128/mBio.00186-17

Lipuma, J. J. (2010). The Changing Microbial Epidemiology in Cystic Fibrosis. Clin. Microbiol. Rev. 23, 299-29+. doi: 10.1128/CMR.00068-09

Luqman, A., Ebner, P., Reichert, S., Sass, P., Kabagema-Bilan, C., Heilmann, C., et al. (2019). A New Host Cell Internalisation Pathway for SadA-Expressing Staphylococci Triggered by Excreted Neurochemicals. Cell Microbiol. 21, e13044. doi: 10.1111/cmi.13044

Luqman, A., Nega, M., Nguyen, M. T., Ebner, P., and Götz, F. (2018). SadAExpressing Staphylococci in the Human Gut Show Increased Cell Adherence and Internalization. Cell Rep. 22, 535-545. doi: 10.1016/j.celrep.2017.12.058

Lyczak, J. B., Cannon, C. L., and Pier, G. B. (2002). Lung Infections Associated With Cystic Fibrosis. Clin. Microbiol. Rev. 15, 194-19+. doi: 10.1128/ CMR.15.2.194-222.2002

Machan, Z. A., Taylor, G. W., Pitt, T. L., Cole, P. J., and Wilson, R. (1992). 2Heptyl-4-Hydroxyquinoline N-Oxide, an Antistaphylococcal Agent Produced by Pseudomonas Aeruginosa. J. Antimicrob. Chemother. 30, 615-623. doi: $10.1093 / \mathrm{jac} / 30.5 .615$

Marques, C. N., Morozov, A., Planzos, P., and Zelaya, H. M. (2014). The Fatty Acid Signaling Molecule Cis-2-Decenoic Acid Increases Metabolic Activity and Reverts Persister Cells to an Antimicrobial-Susceptible State. Appl. Environ. Microbiol. 80, 6976-6991. doi: 10.1128/AEM.01576-14

Mashburn, L. M., Jett, A. M., Akins, D. R., and Whiteley, M. (2005). Staphylococcus Aureus Serves as an Iron Source for Pseudomonas Aeruginosa During In Vivo Coculture. J. Bacteriol. 187, 554-566. doi: 10.1128/JB.187.2.554-566.2005

Michailova, L., Kussovsky, V., Radoucheva, T., Jordanova, M., and Markova, N. (2007). Persistence of Staphylococcus Aureus L-Form During Experimental Lung Infection in Rats. FEMS Microbiol. Lett. 268, 88-97. doi: 10.1111/j.15746968.2006.00567.x

Michelsen, C. F., Christensen, A. M., Bojer, M. S., Hoiby, N., Ingmer, H., and Jelsbak, L. (2014). Staphylococcus Aureus Alters Growth Activity, Autolysis, and Antibiotic Tolerance in a Human Host-Adapted Pseudomonas Aeruginosa Lineage. J. Bacteriol. 196, 3903-3911. doi: 10.1128/JB.02006-14

Millette, G., Langlois, J. P., Brouillette, E., Frost, E. H., Cantin, A. M., and Malouin, F. (2019). Despite Antagonism In Vitro, Pseudomonas Aeruginosa Enhances Staphylococcus Aureus Colonization in a Murine Lung Infection Model. Front. Microbiol. 10, 2880. doi: 10.3389/fmicb.2019.02880

Mitchell, G., Seguin, D. L., Asselin, A. E., Deziel, E., Cantin, A. M., Frost, E. H., et al. (2010). Staphylococcus Aureus Sigma B-Dependent Emergence of SmallColony Variants and Biofilm Production Following Exposure to Pseudomonas Aeruginosa 4-Hydroxy-2-Heptylquinoline-N-Oxide. BMC Microbiol. 10, 33. doi: 10.1186/1471-2180-10-33

Moisan, H., Brouillette, E., Jacob, C. L., Langlois-Begin, P., Michaud, S., and Malouin, F. (2006). Transcription of Virulence Factors in Staphylococcus Aureus Small-Colony Variants Isolated From Cystic Fibrosis Patients is Influenced by SigB. J. Bacteriol. 188, 64-76. doi: 10.1128/JB.188.1.64-76.2006

Morohoshi, T., Kamimura, Y., and Someya, N. (2020). Identification and Characterization of Quorum-Quenching Activity of N-Acylhomoserine Lactonase From Coagulase-Negative Staphylococci. Antibiotics (Basel) 9, 483. doi: 10.3390/antibiotics9080483

Nakayama, K., Takashima, K., Ishihara, H., Shinomiya, T., Kageyama, M., Kanaya, S., et al. (2000). The R-Type Pyocin of Pseudomonas Aeruginosa Is Related to P2 Phage, and the F-Type is Related to Lambda Phage. Mol. Microbiol. 38, 213 231. doi: 10.1046/j.1365-2958.2000.02135.x

Nasir, M., Bean, H. D., Smolinska, A., Rees, C. A., Zemanick, E. T., and Hill, J. E. (2018). Volatile Molecules From Bronchoalveolar Lavage Fluid can 'Rule-In' Pseudomonas Aeruginosa and 'Rule-Out' Staphylococcus Aureus Infections in Cystic Fibrosis Patients. Sci. Rep. 8, 826. doi: 10.1038/s41598-017-18491-8

Noto, M. J., Burns, W. J., Beavers, W. N., and Skaar, E. P. (2017). Mechanisms of Pyocyanin Toxicity and Genetic Determinants of Resistance in Staphylococcus Aureus. J. Bacteriol. 199, e00221-17. doi: 10.1128/JB.00221-17

Orazi, G., and O'toole, G. A. (2017). Pseudomonas Aeruginosa Alters Staphylococcus Aureus Sensitivity to Vancomycin in a Biofilm Model of Cystic Fibrosis Infection. mBio 8, e00873-17. doi: 10.1128/mBio.00873-17

Pagels, M., Fuchs, S., Pane-Farre, J., Kohler, C., Menschner, L., Hecker, M., et al. (2010). Redox Sensing by a Rex-Family Repressor is Involved in the Regulation of Anaerobic Gene Expression in Staphylococcus Aureus. Mol. Microbiol. 76, 1142-1161. doi: 10.1111/j.1365-2958.2010.07105.x 
Painter, K. L., Strange, E., Parkhill, J., Bamford, K. B., Armstrong-James, D., and Edwards, A. M. (2015). Staphylococcus Aureus Adapts to Oxidative Stress by Producing H2O2-Resistant Small-Colony Variants via the SOS Response. Infect. Immun. 83, 1830-1844. doi: 10.1128/IAI.03016-14

Pallett, R., Leslie, L. J., Lambert, P. A., Milic, I., Devitt, A., and Marshall, L. J. (2019). Anaerobiosis Influences Virulence Properties of Pseudomonas Aeruginosa Cystic Fibrosis Isolates and the Interaction With Staphylococcus Aureus. Sci. Rep. 9, 6748. doi: 10.1038/s41598-019-42952-x

Pamp, S. J., and Tolker-Nielsen, T. (2007). Multiple Roles of Biosurfactants in Structural Biofilm Development by Pseudomonas Aeruginosa. J. Bacteriol. 189, 2531-2539. doi: 10.1128/JB.01515-06

Pearson, J. P., Feldman, M., Iglewski, B. H., and Prince, A. (2000). Pseudomonas Aeruginosa Cell-to-Cell Signaling is Required for Virulence in a Model of Acute Pulmonary Infection. Infect. Immun. 68, 4331-4334. doi: 10.1128/ IAI.68.7.4331-4334.2000

Pernet, E., Guillemot, L., Burgel, P. R., Martin, C., Lambeau, G., Sermet-Gaudelus, I., et al. (2014). Pseudomonas Aeruginosa Eradicates Staphylococcus Aureus by Manipulating the Host Immunity. Nat. Commun. 5, 5105. doi: 10.1038/ncomms6105

Pessi, G., and Haas, D. (2000). Transcriptional Control of the Hydrogen Cyanide Biosynthetic Genes honABC by the Anaerobic Regulator ANR and the Quorum-Sensing Regulators LasR and RhlR in Pseudomonas Aeruginosa. J. Bacteriol. 182, 6940-6949. doi: 10.1128/JB.182.24.6940-6949.2000

Pessi, G., and Haas, D. (2001). Dual Control of Hydrogen Cyanide Biosynthesis by the Global Activator GacA in Pseudomonas Aeruginosa PAO1. FEMS Microbiol. Lett. 200, 73-78. doi: 10.1111/j.1574-6968.2001.tb10695.x

Price, C. E., Brown, D. G., Limoli, D. H., Phelan, V. V., and O'toole, G. A. (2020). Exogenous Alginate Protects Staphylococcus Aureus From Killing by Pseudomonas Aeruginosa. J. Bacteriol. 202, e00559-19. doi: 10.1128/JB.00559-19

Qazi, S., Middleton, B., Muharram, S. H., Cockayne, A., Hill, P., O'shea, P., et al. (2006). N-Acylhomoserine Lactones Antagonize Virulence Gene Expression and Quorum Sensing in Staphylococcus Aureus. Infect. Immun. 74, 910-919. doi: 10.1128/IAI.74.2.910-919.2006

Radlinski, L., Rowe, S. E., Kartchner, L. B., Maile, R., Cairns, B. A., Vitko, N. P., et al. (2017). Pseudomonas Aeruginosa Exoproducts Determine Antibiotic Efficacy Against Staphylococcus Aureus. PloS Biol. 15, e2003981. doi: 10.1371/journal.pbio.2003981

Schäfer, D., Lam, T. T., Geiger, T., Mainiero, M., Engelmann, S., Hussain, M., et al. (2009). A Point Mutation in the Sensor Histidine Kinase SaeS of Staphylococcus Aureus Strain Newman Alters the Response to Biocide Exposure. J. Bacteriol. 191, 7306-7314. doi: 10.1128/JB.00630-09

Schmidt, K. A., Manna, A. C., and Cheung, A. L. (2003). SarT Influences sarS Expression in Staphylococcus Aureus. Infect. Immun. 71, 5139-5148. doi: 10.1128/IAI.71.9.5139-5148.2003

Schmitt-Slomska, J., Michailova, L., Ivanova, E., and Toshkov, A. (1986). Adhesion and Phagocytosis of Staphylococcus Aureus L-Forms. J. Basic Microbiol. 26, 429-440. doi: 10.1002/jobm.3620260712

Simonin, J., Bille, E., Crambert, G., Noel, S., Dreano, E., Edwards, A., et al. (2019). Airway Surface Liquid Acidification Initiates Host Defense Abnormalities in Cystic Fibrosis. Sci. Rep. 9, 6516. doi: 10.1038/s41598-019-42751-4

Smith, A. C., Rice, A., Sutton, B., Gabrilska, R., Wessel, A. K., Whiteley, M., et al. (2017). Albumin Inhibits Pseudomonas Aeruginosa Quorum Sensing and Alters Polymicrobial Interactions. Infect. Immun. 85, e00116-17. doi: 10.1128/IAI.00116-17

Szamosvari, D., and Böttcher, T. (2017). An Unsaturated Quinolone N-Oxide of Pseudomonas Aeruginosa Modulates Growth and Virulence of Staphylococcus Aureus. Angew. Chem. Int. Ed. Engl. 56, 7271-7275. doi: 10.1002/ anie. 201702944

Tamber, S., and Cheung, A. L. (2009). SarZ Promotes the Expression of Virulence Factors and Represses Biofilm Formation by Modulating SarA and Agr in Staphylococcus Aureus. Infect. Immun. 77, 419-428. doi: 10.1128/IAI.00859-08
Tognon, M., Kohler, T., Gdaniec, B. G., Hao, Y., Lam, J. S., Beaume, M., et al. (2017). Co-Evolution With Staphylococcus Aureus Leads to Lipopolysaccharide Alterations in Pseudomonas Aeruginosa. ISME J. 11, 2233-2243. doi: 10.1038/ ismej. 2017.83

Tognon, M., Kohler, T., Luscher, A., and Van Delden, C. (2019). Transcriptional Profiling of Pseudomonas Aeruginosa and Staphylococcus Aureus During In Vitro Co-Culture. BMC Genomics 20, 30. doi: 10.1186/s12864-018-5398-y

Trizna, E. Y., Yarullina, M. N., Baidamshina, D. R., Mironova, A. V., Akhatova, F. S., Rozhina, E. V., et al. (2020). Bidirectional Alterations in Antibiotics Susceptibility in Staphylococcus Aureus-Pseudomonas Aeruginosa DualSpecies Biofilm. Sci. Rep. 10, 14849. doi: 10.1038/s41598-020-71834-w

Van Delden, C., and Iglewski, B. H. (1998). Cell-To-Cell Signaling and Pseudomonas Aeruginosa Infections. Emerg. Infect. Dis. 4, 551-560. doi: 10.3201/eid0404.980405

Verkman, A. S., Song, Y., and Thiagarajah, J. R. (2003). Role of Airway Surface Liquid and Submucosal Glands in Cystic Fibrosis Lung Disease. Am. J. Physiol. Cell Physiol. 284, C2-15. doi: 10.1152/ajpcell.00417.2002

Voggu, L., Schlag, S., Biswas, R., Rosenstein, R., Rausch, C., and Götz, F. (2006). Microevolution of Cytochrome $B d$ Oxidase in Staphylococci and its Implication in Resistance to Respiratory Toxins Released by Pseudomonas. J. Bacteriol. 188, 8079-8086. doi: 10.1128/JB.00858-06

Wakeman, C. A., Moore, J. L., Noto, M. J., Zhang, Y., Singleton, M. D., Prentice, B. M., et al. (2016). The Innate Immune Protein Calprotectin Promotes Pseudomonas Aeruginosa and Staphylococcus Aureus Interaction. Nat. Commun. 7, 11951. doi: 10.1038/ncomms11951

Welsh, M. A., Eibergen, N. R., Moore, J. D., and Blackwell, H. E. (2015). Small Molecule Disruption of Quorum Sensing Cross-Regulation in Pseudomonas Aeruginosa Causes Major and Unexpected Alterations to Virulence Phenotypes. J. Am. Chem. Soc. 137, 1510-1519. doi: 10.1021/ja5110798

Williams, B. J., Dehnbostel, J., and Blackwell, T. S. (2010). Pseudomonas Aeruginosa: Host Defence in Lung Diseases. Respirology 15, 1037-1056. doi: 10.1111/j.1440-1843.2010.01819.x

Wilson, R., Sykes, D. A., Watson, D., Rutman, A., Taylor, G. W., and Cole, P. J. (1988). Measurement of Pseudomonas Aeruginosa Phenazine Pigments in Sputum and Assessment of Their Contribution to Sputum Sol Toxicity for Respiratory Epithelium. Infect. Immun. 56, 2515-2517. doi: 10.1128/ iai.56.9.2515-2517.1988

Wood, T. L., Gong, T., Zhu, L., Miller, J., Miller, D. S., Yin, B., et al. (2018). Rhamnolipids From Pseudomonas Aeruginosa Disperse the Biofilms of SulfateReducing Bacteria. NPJ Biofilms Microbiomes 4, 22. doi: 10.1038/s41522-0180066-1

Conflict of Interest: The authors declare that the research was conducted in the absence of any commercial or financial relationships that could be construed as a potential conflict of interest.

Publisher's Note: All claims expressed in this article are solely those of the authors and do not necessarily represent those of their affiliated organizations, or those of the publisher, the editors and the reviewers. Any product that may be evaluated in this article, or claim that may be made by its manufacturer, is not guaranteed or endorsed by the publisher.

Copyright $\odot 2022$ Biswas and Götz. This is an open-access article distributed under the terms of the Creative Commons Attribution License (CC BY). The use, distribution or reproduction in other forums is permitted, provided the original author $(s)$ and the copyright owner(s) are credited and that the original publication in this journal is cited, in accordance with accepted academic practice. No use, distribution or reproduction is permitted which does not comply with these terms. 